\title{
Spin-resolved bunching and noise characteristics in double quantum dots coupled to ferromagnetic electrodes
}

\author{
JunYan Luo, , * HuJun Jiao, ${ }^{2}$ BiTao Xiong, ${ }^{1}$ Xiao-Ling He, ${ }^{1}$ and Changrong Wang ${ }^{1}$ \\ ${ }^{1}$ School of Science, Zhejiang University of Science and Technology, Hangzhou 310023, China \\ ${ }^{2}$ Department of Physics, Shanxi University, Taiyuan, Shanxi 030006, China
}

(Dated: November 13, 2018)

\begin{abstract}
We study spin-resolved noise in Coulomb blockaded double quantum dots coupled to ferromagnetic electrodes. The modulation of the interdot coupling and spin polarization in the electrodes gives rise to an intriguing dynamical spin $\uparrow-\uparrow(\downarrow-\downarrow)$ blockade mechanism: Bunching of up (down) spins due to dynamical blockade of an up (down) spin. In contrast to the conventional dynamical spin $\uparrow-\downarrow$ bunching (bunching of up spins entailed by dynamical blockade of a down spin), this new bunching behavior is found to be intimately associated with the spin mutual-correlation, i.e., the noise fluctuation between opposite spin currents. We further demonstrate that the dynamical spin $\uparrow-\uparrow$ and $\uparrow-\downarrow$ bunching of tunneling events may be coexistent in the regime of weak interdot coupling and low spin polarization.
\end{abstract}

PACS numbers: 72.25.-b, 72.70.+m, 73.63.Kv, 73.23.Hk

\section{INTRODUCTION}

The measurement of signal-to-noise ratio in mesoscopic transport devices is of vital importance as it enables access to intriguing information about the statistics of quasiparticles and various intrinsic dynamics that are not available from conventional current measurements alone [1, 2]. For transport through a localized state, the nonequilibrium noise is generally suppressed due to the Pauli exclusion principle, leading thus to a subPoissonian statistics [3-5]. However, in systems of multiple nonlocal states, such as double quantum dot devices, the intrinsic quantum coherence and many-particle interactions give rise to different sources of correlations [6]. Electron transport can exhibit a unique dynamical blockade mechanism, leading thus to a super-Poisson fluctuation $7-12$.

In spintronic structures, transport is governed not only by the charge flow, but more importantly, by the spin transfer [13 20]. The involving spin degrees of freedom introduce additional correlated mechanisms. Study of spin current fluctuations is crucial for possible applications in control and manipulation of individual spins. A number of investigations have been devoted to the noise characteristics of spin-dependent transport through various nanostructures, such as quantum dots [19 22], molecules [23 25], and nanotubes 26 29]. Different tunneling processes like sequential tunneling, cotunneling [30, 31], etc. were revealed to have vital roles to play in spin transport. In order to distinguish various spin dynamics it is instructive to unravel the charge noise into spin-resolved components. Consider a general mesoscopic device of a quantum dot (QD) system connected to terminals $\alpha, \alpha^{\prime} \ldots$. The charge current through the ter-

*Electronic address: jyluo@zust.edu.cn

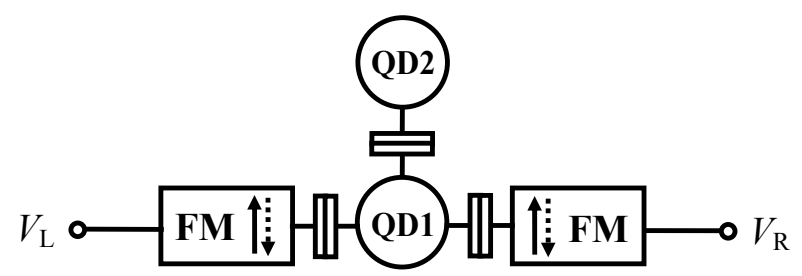

FIG. 1: Schematic setup for spin-dependent transport through double quantum dots, in which only QD1 is tunnelcoupled to ferromagnetic electrodes.

minal " $\alpha$ " is $\left\langle I_{\alpha}\right\rangle=\left\langle I_{\alpha}^{\sigma}\right\rangle+\left\langle I_{\alpha}^{-\sigma}\right\rangle$, where $\left\langle I_{\alpha}^{\sigma}\right\rangle$ is the spin- $\sigma$ component of the current. The temporal correlation of spin transport is characterized by the spin-resolved correlation function $C_{\alpha \alpha^{\prime}}^{\sigma \sigma^{\prime}}\left(t-t^{\prime}\right)=\frac{1}{2}\left\langle\left\{\Delta I_{\alpha}^{\sigma}(t), \Delta I_{\alpha^{\prime}}^{\sigma^{\prime}}\left(t^{\prime}\right)\right\}\right\rangle$, with $\Delta I_{\alpha}^{\sigma}(t)=I_{\alpha}^{\sigma}(t)-\left\langle I_{\alpha}^{\sigma}(t)\right\rangle$. Straightforwardly, the individual spin-resolved noise power is given by $S_{\alpha \alpha^{\prime}}^{\sigma \sigma^{\prime}}(\omega)=$ $\int_{-\infty}^{\infty} \mathrm{d} t e^{\mathrm{i} \omega t} C_{\alpha \alpha^{\prime}}^{\sigma \sigma^{\prime}}(t)$. By choosing an arbitrary spin axis $\mathbf{z}$, the total charge current noise can be readily unraveled into $S_{\alpha \alpha^{\prime}}^{\mathrm{ch}}=S_{\alpha \alpha^{\prime}}^{\uparrow \uparrow}+S_{\alpha \alpha^{\prime}}^{\downarrow \downarrow}+S_{\alpha \alpha^{\prime}}^{\uparrow \downarrow}+S_{\alpha \alpha^{\prime}}^{\downarrow \uparrow}$. Here, the spin self-correlation $S_{\alpha \alpha^{\prime}}^{\uparrow \uparrow}\left(S_{\alpha \alpha^{\prime}}^{\downarrow \downarrow}\right)$ represents fluctuation between the same spin currents, which was recently shown to be capable of serving as a sensitive tool to identify the dynamical spin $\uparrow-\downarrow(\downarrow-\uparrow)$ bunching, i.e., bunching of up (down) spins due to dynamical blockade of a down (up) spin 32 34]. It then naturally comes to a question: What can we infer from the spin mutual-correlation noise (fluctuation between opposite spin currents) $S_{\alpha \alpha^{\prime}}^{\uparrow \downarrow}$ or $S_{\alpha \alpha^{\prime}}^{\downarrow \uparrow}$ ?

In the context of spin-dependent transport through a system of multiple nonlocal states, we investigate in this work these spin-resolved noise components and their connections to spin-resolved bunching behavior. Specifically, we consider a double quantum dot, where only one of the dots is tunnel-coupled to the ferromagnetic (FM) elec- 

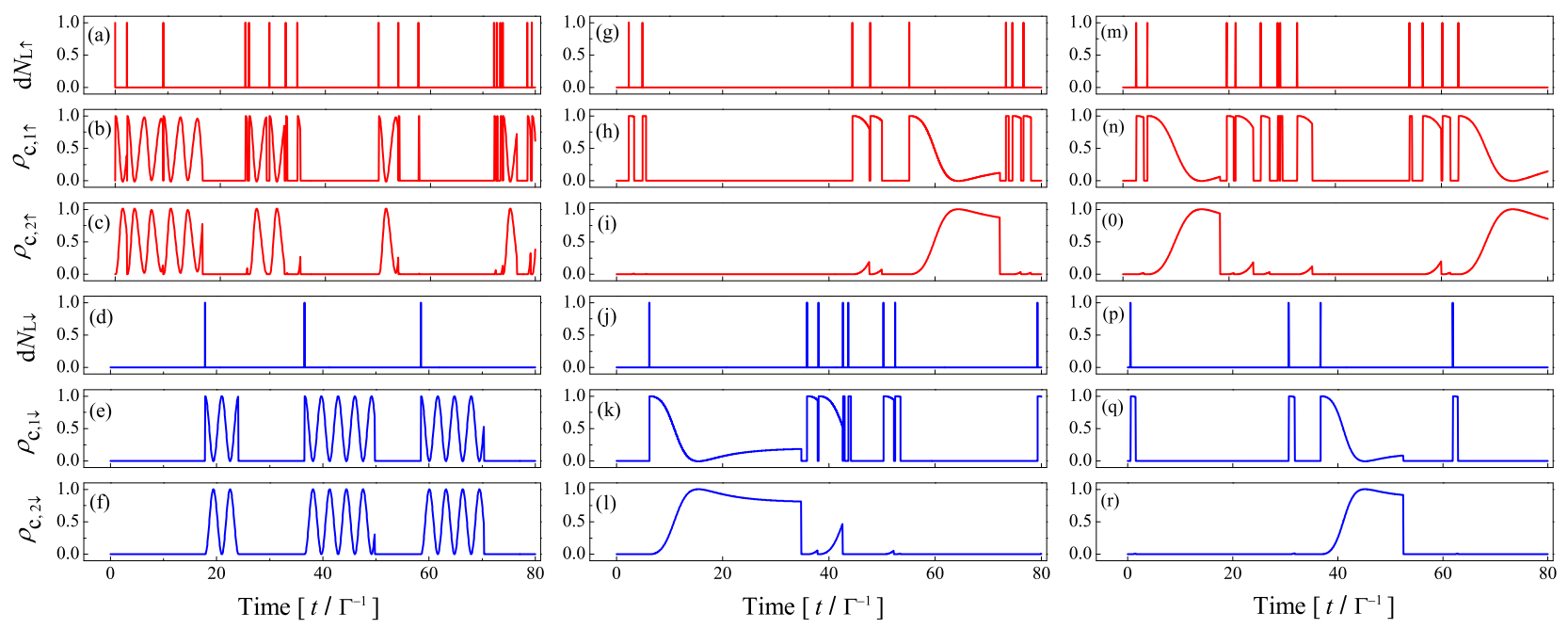

FIG. 2: Sets of typical detection record (up and down spin tunneling events) and corresponding real-time quantum states under parallel magnetic alignment. (a)-(f) $\Omega / \Gamma=1.0$ and $p=0.8$, (g)-(l) $\Omega / \Gamma=0.2$ and $p=0$, and $(\mathrm{m})-(\mathrm{r}) \Omega / \Gamma=0.1$ and $p=0.25$. Each quantum dot has only one level (assumed to be in resonance, i.e., $E_{1}=E_{2}$ ) within the bias window $V=V_{\mathrm{L}}-V_{\mathrm{R}}$. In the Coulomb blockade regime and for temperature $k_{\mathrm{B}} T \ll V$, the Fermi functions can be approximated by either one or zero, so the temperature is not involved here.

trodes (see Fig.11). The system, which can be mapped to the one investigated recently in experiments [35 38 , is of particular interest, as it is arranged in such a configuration that can maximize locality versus nonlocality contrast 39 42. In the Coulomb blockade regime, we observe a unique dynamical spin $\uparrow-\uparrow(\downarrow-\downarrow)$ blockade phenomenon, namely, bunching of up (down) spins due to dynamical blockade of an up (down) spin. Different from the conventional spin $\uparrow-\downarrow$ bunching, it is revealed that this new mechanism is intimately associated with positive spin mutual-correlation. We further demonstrate that the spin $\uparrow-\uparrow$ and $\uparrow-\downarrow$ bunching of tunneling events may be coexistent in the regime of low spin polarization and weak interdot tunnel-coupling.

The paper is organized as follows. In Sec.II] we describe the double quantum dot system tunnel-coupled to FM electrodes. In Sec.III a Monte Carlo approach is introduced to simulate real-time single spin tunneling events. The spin-resolved noises, together with spinresolved bunching of tunneling events will be discussed in detail. It is then followed by the conclude in Sec.IV.

\section{MODEL DESCRIPTION}

The system under study is sketched in Fig.1, in which only QD1 is connected to the FM electrodes, whereas QD2 is side-connected to the QD1. The Hamiltonian of the entire system is $\hat{H}=\hat{H}_{\mathrm{B}}+\hat{H}_{\mathrm{S}}+\hat{H}^{\prime}$, where $\hat{H}_{\mathrm{B}}=\sum_{\alpha=\mathrm{L}, \mathrm{R}} \sum_{k, \sigma} \varepsilon_{\alpha k \sigma} c_{\alpha k \sigma}^{\dagger} c_{\alpha k \sigma}$ denotes the left and right FM electrodes; $c_{\alpha k \sigma}\left(c_{\alpha k \sigma}^{\dagger}\right)$ is the electron annihilation (creation) operator of the electrode $\alpha=\mathrm{L}$ or $\mathrm{R}$, with spin $\sigma=\uparrow$ or $\downarrow$. The ferromagnetism of the electrode $\alpha$ is accounted for by the spin-dependent density of states $g_{\alpha \sigma}(\omega)$. Throughout all of our calculations presented here, we approximate the density of states to be energy independent, $g_{\alpha \sigma}(\omega)=g_{\alpha \sigma}$. (Real ferromagnets have a structured density of states, which only modifies details of our results but not the general physical picture.) The asymmetry in the density of states is characterized by the degree of spin polarization $p_{\alpha}=\left(g_{\alpha \uparrow}-g_{\alpha \downarrow}\right) /\left(g_{\alpha \uparrow}+g_{\alpha \downarrow}\right)$ with $-1 \leq p_{\alpha} \leq 1$, in which $p_{\alpha}=0$ denotes a nonmagnetic electrode and $p_{\alpha}= \pm 1$ corresponds to a halfmetallic electrode.

The Hamiltonian for the coupled dots reads

$$
\begin{aligned}
\hat{H}_{\mathrm{S}}= & \sum_{\ell=1,2}\left[\sum_{\sigma=\uparrow, \downarrow} E_{\ell} \hat{n}_{\ell \sigma}+U_{0} \hat{n}_{\ell \uparrow} \hat{n}_{\ell \downarrow}\right]+U^{\prime} \hat{n}_{1} \hat{n}_{2} \\
& +\Omega \sum_{\sigma}\left(d_{1 \sigma}^{\dagger} d_{2 \sigma}+d_{2 \sigma}^{\dagger} d_{1 \sigma}\right)
\end{aligned}
$$

where $d_{\ell \sigma}\left(d_{\ell \sigma}^{\dagger}\right)$ is the creation (annihilation) operator of an electron with spin $\sigma=\uparrow$ or $\downarrow$ in $\mathrm{QD} 1(\ell=1)$ or QD2 $(\ell=2) ; \hat{n}_{\ell \sigma}=d_{\ell \sigma}^{\dagger} d_{\ell \sigma}$ and $\hat{n}_{\ell}=\sum_{\sigma} \hat{n}_{\ell \sigma}$ are the corresponding particle number operators. Each QD has a spin-degenerate level within the bias window $V=$ $V_{\mathrm{L}}-V_{\mathrm{R}} \cdot U_{0}$ and $U^{\prime}$ are respectively the intradot and interdot Coulomb repulsions; $\Omega$ denotes the strength of interdot tunnel-coupling. Hereafter, we consider doubledot Coulomb blockade regime [43, 44], i.e., $U_{0}$ and $U^{\prime}$ are large enough such that states with two or more electrons in the double dots are not allowed. The involved states are both dots empty $|0\rangle$, one electron with spin $\sigma$ in the 

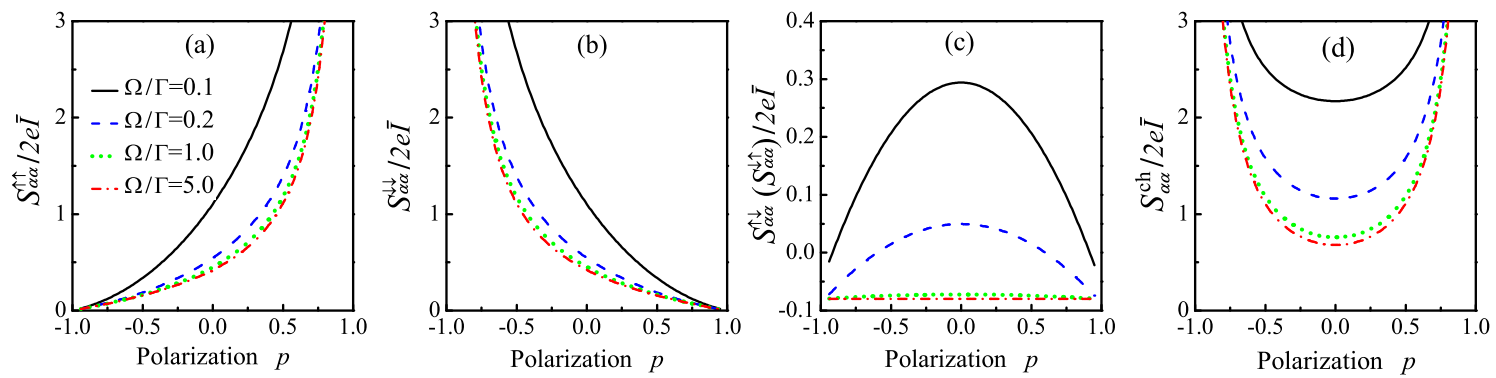

FIG. 3: Spin self-correlations $S_{\alpha \alpha}^{\uparrow \uparrow}$ and $S_{\alpha \alpha}^{\downarrow \downarrow}$, spin mutual-correlation $S_{\alpha \alpha}^{\uparrow \downarrow}\left(S_{\alpha \alpha}^{\downarrow \uparrow}\right)$, as well as the total charge current noise $S_{\alpha \alpha}^{\text {ch }}$ vs polarization $p$ for various interdot couplings $\Omega / \Gamma=0.1,0.2,1.0,5.0$. The left and right electrodes are of $\mathrm{P}$ configuration. The other parameters are the same as those in Fig.2

QD1 $|1 \sigma\rangle$ or QD2 $|2 \sigma\rangle$. Experimentally, it can be realized by properly tuning the gate and bias voltages [6, 45 47].

Tunneling between QD1 and electrodes is described by $\hat{H}^{\prime}=\sum_{\alpha k \sigma}\left(t_{\alpha k \sigma} c_{\alpha k \sigma}^{\dagger} d_{1 \sigma}+\right.$ H.c. $)$. The FM electrodes result in spin-dependent tunnel couplings $\Gamma_{\alpha \sigma}=$ $2 \pi \sum_{k}\left|t_{\alpha k \sigma}\right|^{2} \delta\left(\varepsilon_{\alpha k \sigma}-\omega\right)$. In what follows, we consider two magnetic configurations. (i) The parallel (P) alignment, when the majority of electrons in both electrodes point in the same direction. (ii) The antiparallel (AP) case, in which the magnetization of the two electrodes are opposite to each other. Thus for the $\mathrm{P}$ alignment we have

$$
\Gamma_{\mathrm{L} \uparrow / \mathrm{L} \downarrow}=\frac{1}{2}\left(1 \pm p_{\mathrm{L}}\right) \Gamma_{\mathrm{L}} \text { and } \Gamma_{\mathrm{R} \uparrow / \mathrm{R} \downarrow}=\frac{1}{2}\left(1 \pm p_{\mathrm{R}}\right) \Gamma_{\mathrm{R}},
$$

while for the AP configuration we set

$$
\Gamma_{\mathrm{L} \uparrow / \mathrm{L} \downarrow}=\frac{1}{2}\left(1 \pm p_{\mathrm{L}}\right) \Gamma_{\mathrm{L}} \text { and } \Gamma_{\mathrm{R} \uparrow / \mathrm{R} \downarrow}=\frac{1}{2}\left(1 \mp p_{\mathrm{R}}\right) \Gamma_{\mathrm{R}} .
$$

Here $\Gamma_{\alpha}=\left(\Gamma_{\alpha \uparrow}+\Gamma_{\alpha \downarrow}\right)$ is the total coupling strength regardless the spin orientation.

\section{RESULTS AND DISCUSSIONS}

In order to get a deep understanding of the spin dynamics and fluctuations in transport, a Monte Carlo method is employed to simulate the real-time single spin tunneling events in the quantum-jump regime. We introduce two stochastic point variables $\mathrm{d} N_{\mathrm{L} \sigma}(t)$ and $\mathrm{d} N_{\mathrm{R} \sigma}(t)$ (with values either 0 or 1 ) to stand for, respectively, the numbers of spin- $\sigma(\sigma=\uparrow, \downarrow)$ electron tunneled to the double dots from left electrode and that from the double dots to the right electrode, during the time interval $\mathrm{d} t$. The conditional quantum master equation for the reduced density matrix reads [48]

$$
\begin{aligned}
\mathrm{d} \rho_{\mathrm{c}}=-\mathrm{i} \mathcal{L} \rho_{\mathrm{c}}(t) \mathrm{d} t-\sum_{\sigma=\uparrow, \downarrow}\left\{\Gamma_{\mathrm{L} \sigma} \mathcal{A}\left[d_{1 \sigma}^{\dagger}\right]+\Gamma_{\mathrm{R} \sigma} \mathcal{A}\left[d_{1 \sigma}\right]\right. \\
\left.-\mathcal{P}_{\mathrm{L} \sigma}(t)-\mathcal{P}_{\mathrm{R} \sigma}(t)\right\} \rho_{\mathrm{c}}(t) \mathrm{d} t \\
+\sum_{\sigma=\uparrow, \downarrow} \mathrm{d} N_{\mathrm{L} \sigma}\left[\frac{\Gamma_{\mathrm{L} \sigma} \mathcal{J}\left[d_{1 \sigma}^{\dagger}\right]}{\mathcal{P}_{\mathrm{L} \sigma}(t)}-1\right] \rho_{\mathrm{c}}(t) \\
+\sum_{\sigma=\uparrow, \downarrow} \mathrm{d} N_{\mathrm{R} \sigma}\left[\frac{\Gamma_{\mathrm{R} \sigma} \mathcal{J}\left[d_{1 \sigma}\right]}{\mathcal{P}_{\mathrm{R} \sigma}(t)}-1\right] \rho_{\mathrm{c}}(t),
\end{aligned}
$$

where the attached subscript "c" is to indicate that the quantum state is conditioned on previous measurement results. Here the superoperators are defined as $\mathcal{L}(\cdots) \equiv$ $\left[\hat{H}_{\mathrm{S}}, \cdots\right], \mathcal{J}[X] \rho_{\mathrm{c}} \equiv X \rho_{\mathrm{c}} X^{\dagger}$ and $\mathcal{A}[X] \rho_{\mathrm{c}} \equiv \frac{1}{2}\left(X^{\dagger} X \rho_{\mathrm{c}}+\right.$ $\left.\rho_{\mathrm{c}} X^{\dagger} X\right)$. The involving stochastic point variables satisfy

$$
\begin{aligned}
& E\left[\mathrm{~d} N_{\mathrm{L} \sigma}(t)\right]=\mathcal{P}_{\mathrm{L} \sigma}(t) \mathrm{d} t=\operatorname{Tr}\left\{\mathcal{J}\left[\sqrt{\Gamma_{\mathrm{L} \sigma}} d_{1 \sigma}^{\dagger}\right] \rho_{\mathrm{c}}\right\} \mathrm{d} t,(4 \mathrm{a}) \\
& E\left[\mathrm{~d} N_{\mathrm{R} \sigma}(t)\right]=\mathcal{P}_{\mathrm{R} \sigma}(t) \mathrm{d} t=\operatorname{Tr}\left\{\mathcal{J}\left[\sqrt{\Gamma_{\mathrm{R} \sigma}} d_{1 \sigma}\right] \rho_{\mathrm{c}}\right\} \mathrm{d} t,(4 \mathrm{~b})
\end{aligned}
$$

where $E[(\cdots)]$ denotes an ensemble average of a large number of quantum trajectories. In this quantum trajectory approach, spin tunneling events condition the future evolution of the system state [see Eq. (3)], while the instantaneous quantum state conditions the measured spin tunneling events through the double dots [see Eq. (4a) and Eq. (4b)] in a self-consistent manner. One thus is propagating in parallel the information of the conditioned (stochastic) state evolution $\left(\rho_{\mathrm{c}}\right)$ and detection record $\left(\mathrm{d} N_{\alpha \sigma}\right)$ in a single realization of the readout measurement experiment.

The spin tunneling events $\left(\mathrm{d} N_{\alpha \sigma}\right)$ leads straightforwardly to the spin- $\sigma$ dependent current $I_{\alpha}^{\sigma}(t)=$ $e \mathrm{~d} N_{\alpha \sigma}(t) / \mathrm{d} t$, and consequently the total charge current $I_{\alpha}^{\mathrm{ch}}=I_{\alpha}^{\uparrow}+I_{\alpha}^{\downarrow}$. Hereafter, we will use $\bar{I} \equiv E\left[I_{\alpha}^{\mathrm{ch}}(t)\right]_{t \rightarrow \infty}$ to represent the ensemble stationary current. The spinresolved time correlation function $\left[C_{\alpha \alpha^{\prime}}^{\sigma \sigma^{\prime}}(t)\right]$ and its corresponding noise spectrum $\left[S_{\alpha \alpha^{\prime}}^{\sigma \sigma^{\prime}} \equiv S_{\alpha \alpha^{\prime}}^{\sigma \sigma^{\prime}}(\omega=0)\right]$ can be evaluated following [49], or by using alternatively a spinresolved quantum master equation approach [50, 51]. In what follows, noise between different electrodes are not considered as it simply satisfies $S_{\mathrm{LR}}^{\sigma \sigma^{\prime}}=-S_{\alpha \alpha}^{\sigma \sigma^{\prime}}$ for the present two-terminal device (Note such a relation generally does not hold for a multi-terminal structure [32, 33, 52]). For simplicity, we assume symmetric tunnel couplings $\left(\Gamma_{\mathrm{L}}=\Gamma_{\mathrm{R}}=\Gamma / 2\right)$ and equal magnitude of spin polarization in the two electrodes $\left(p_{\mathrm{L}}=p_{\mathrm{R}}=p\right)$.

First let us focus on the $\mathrm{P}$ alignment. Fig.2(a)-(f) show the set of measured spin tunneling events $\left(\mathrm{d} N_{\mathrm{L} \uparrow / \mathrm{L} \downarrow}\right)$ and the corresponding real-time quantum state $\left(\rho_{\mathrm{c}}\right)$ for polarization $p=0.8$ and interdot coupling $\Omega / \Gamma=1.0$. We observe unambiguously the bunching of up-spin tunneling events. When a down spin is injected into the double 
dots, it will stay there and experiences some oscillations between the two dots, until it finally escapes to the right electrode [see Fig.2 (e) and (f) the instantaneous quantum states of a down spin in QD1 and QD2]. The up spins can flow only in short time windows where the current is not blockaded by a down spin [see Fig.2(a)-(c)], leading thus to the conventional dynamical spin blockade, as discussed in Refs. 32 34]. For clarity, we refer to this mechanism as dynamical spin $\uparrow-\downarrow$ blockade to specify the bunching of up spins due to dynamical blockade of a down spin. The dynamical spin $\uparrow-\downarrow$ blockade gives rise to a prominent super-Poisson spin self-correlation $S_{\alpha \alpha}^{\uparrow \uparrow}$, as shown in Fig.[3(a). Note here we only need to consider $S_{\alpha \alpha}^{\uparrow \uparrow}$ due to the fact that $S_{\alpha \alpha}^{\uparrow \uparrow}$ and $S_{\alpha \alpha}^{\downarrow \downarrow}$ are symmetric with respect to the spin polarization $p$, i.e., Fig.3(a) vs (b).

The spin self-correlation $S_{\alpha \alpha}^{\uparrow \uparrow}$ increases monotonically with the polarization as displayed in Fig.3(a). Only for sufficient spin polarization that the " $\uparrow-\downarrow$ " competition mechanism takes place, which leads eventually to the super-Poisson spin self-correlation $S_{\alpha \alpha}^{\uparrow \uparrow}$. Yet, it is also instructive to investigate the noise at low polarization, for instance $p=0$. The total charge current noise $S_{\alpha \alpha}^{\text {ch }}$ as shown in Fig. 3(d) exhibits unambiguously superPoisson statistics for $\Omega / \Gamma=0.2$ (dashed line). It implies bunching of charge tunneling events regardless of the spin orientations. However, neither of its components $\left(S_{\alpha \alpha}^{\uparrow \uparrow}\right.$ or $\left.S_{\alpha \alpha}^{\downarrow \downarrow}\right)$ exceeds the Poisson value $\left[S_{\alpha \alpha}^{\uparrow \uparrow} /\left.(2 e \bar{I})\right|_{p=0}=\right.$ $\left.S_{\alpha \alpha}^{\downarrow \downarrow} /\left.(2 e \bar{I})\right|_{p=0}=0.46\right]$. It means that the super-Poisson charge noise does not stem from the dynamical spin $\uparrow-\downarrow$ or $\downarrow-\uparrow$ bunching. In other words, the spin self-correlations $S_{\alpha \alpha}^{\uparrow \uparrow}$ and $S_{\alpha \alpha}^{\downarrow \downarrow}$ do not capture the whole picture of spin bunching.

We ascribe the occurrence of the super-Poisson charge noise to a unique dynamical spin $\uparrow-\uparrow$ or $\downarrow-\downarrow$ bunching, which is confirmed by the numerical simulation of realtime spin tunneling as shown in Fig.2(g)-(j) for $p=0$ and $\Omega / \Gamma=0.2$. When a down spin is injected into QD1, it cycles to the QD2, where it is localized due to weak interdot tunnel-coupling. The current is thus blockaded until the down spin finally tunnels to the right electrode, which is then followed by a bunch of down spins flowing through the system, i.e., bunching of down spins due to dynamical blockade of a down spin [see Fig.2(2(k)-(l)]. We call this new mechanism the dynamical spin $\downarrow-\downarrow$ blockade to distinguish it from the $\uparrow-\downarrow$ one. Similarly, dynamical spin $\uparrow-\uparrow$ bunching is observed as shown in Fig.2( $(\mathrm{g})-$ (i). However, the dynamical spin $\uparrow-\uparrow$ or $\downarrow-\downarrow$ blockade does not necessarily give rise to super-Poissonian spin self-correlations. We will reveal this new spin bunching mechanism is intimately associated with the spin mutualcorrelation $S_{\alpha \alpha}^{\uparrow \downarrow}$, which thus can be utilized as an additional diagnostic tool to the dynamics and fluctuations in spin transport.

For a quantitative analysis, we first evaluate some fundamental time scales involved in transport (for $\Omega / \Gamma=0.2$ and $p=0$ ). By using 2000 independent trajectories analogous to the ones shown in Fig.2(g)-(l), we get the average delay between the occupancy of the dots by two consecu-

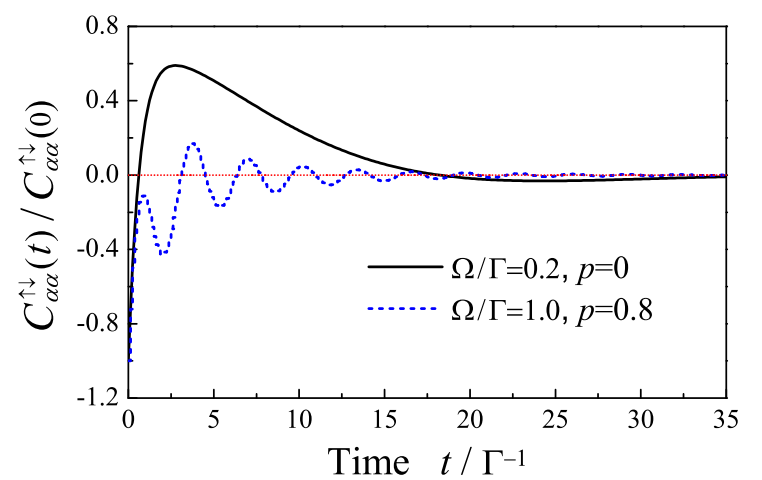

FIG. 4: Time dependence of correlation function $C_{\alpha \alpha}^{\uparrow \downarrow}(t)$ for $\Omega / \Gamma=0.2, p=0$ (solid line) and $\Omega / \Gamma=1.0, p=0.8$ (dotted line).

tive up spins $\tau_{0}=1.01 \Gamma^{-1}$ and the average dwell time of up spins on the double dots $\tau_{\uparrow}=4.00 \Gamma^{-1}$. The average duration of the "bunch" of up spins is then obtained as $\tau_{\mathrm{b}}=6.01 \Gamma^{-1}$. (An alternative approach to obtain these quantities can be found in Ref. 32].) The above time scales are able to reveal the intrinsic dynamics in spin transport. Consider, for instance, the spin-resolved time correlation function $C_{\alpha \alpha}^{\uparrow \downarrow}(t)$, as shown by the solid line in Fig.4. It is negative for times shorter than $\tau_{0}$. It then rises, becomes positive and reaches a maximum at a time comparable to $\tau_{\uparrow}$. Finally, it decreases on a time scale of $\tau_{\uparrow}+\tau_{\mathrm{b}}$. For the time scales of tunneling of down spins, analogous analysis can be applied. The above characteristic times in the correlation function thus allow us to attribute the positive $S_{\alpha \alpha}^{\uparrow \downarrow}$ to the dynamical spin $\uparrow-\uparrow$ or $\downarrow$ $\downarrow$ blockade mechanism. In comparison, these unique time features can not be identified in the case of large interdot coupling $(\Omega / \Gamma=1.0)$ and strong magnetic polarization $(p=0.8)$, as shown by the dotted line in Fig.4.

The requirements to observe the dynamical spin $\uparrow-\uparrow$ or $\downarrow-\downarrow$ bunching of tunneling events thus can be inferred from the spin mutual-correlation. For the P alignment, the analytic expression is give by

$$
S_{\alpha \alpha}^{\uparrow \downarrow}=2 e \bar{I} \frac{\left(1-p^{2}\right) \Gamma^{2}-16 \Omega^{2}}{200 \Omega^{2}} .
$$

It might be either positive or negative, depending on the degree of spin polarization and the strength of interdot tunnel-coupling. For $\Omega<\frac{1}{4} \Gamma$, positive spin mutualcorrelation is observed provided the electrodes are weakly polarized, implying thus the occurrence of dynamical spin $\uparrow-\uparrow$ or $\downarrow-\downarrow$ bunching [cf. Fig.[2(g)-(l)]. It is worth noting that the presented spin transport behavior persists over a wide range of polarization as long as the interdot coupling is weak enough. It thus offers an opportunity to observe the coexistence of spin $\uparrow-\uparrow$ and $\uparrow-\downarrow$ bunching of tunneling events, as displayed in Fig.2(m)-(r) for spin polarization $p=0.25$ and interdot coupling $\Omega / \Gamma=0.1$. In the opposite regime of $\Omega>\frac{1}{4} \Gamma$, the interdot coupling is 

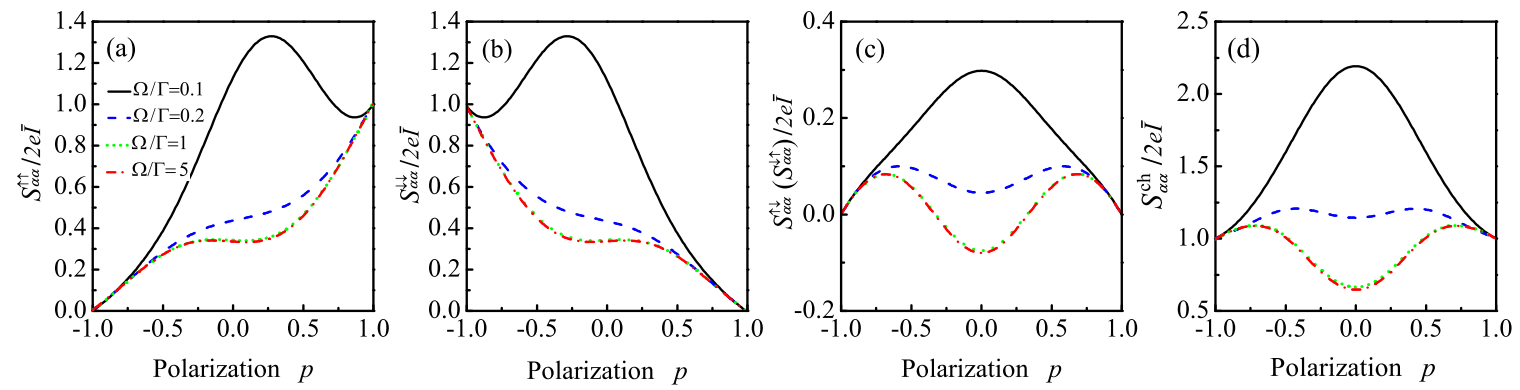

FIG. 5: Spin self-correlations $S_{\alpha \alpha}^{\uparrow \uparrow}$ and $S_{\alpha \alpha}^{\downarrow \downarrow}$, spin mutual-correlation $S_{\alpha \alpha}^{\uparrow \downarrow}\left(S_{\alpha \alpha}^{\downarrow \uparrow}\right)$, as well as the total charge current noise $S_{\alpha \alpha}^{\mathrm{ch}}$ vs polarization $p$ for various interdot couplings $\Omega / \Gamma=0.1,0.2,1.0$, and 5.0. The left and right electrodes are of AP alignment. The other parameters are the same as those in Fig.2

large enough to overcome electron localization in QD2, leading thus to the disappearance of the dynamical spin $\uparrow-\uparrow$ or $\downarrow-\downarrow$ bunching, as confirmed by our numerical realtime simulation (not shown explicitly). The resultant spin mutual-correlation is negative at arbitrary strength of spin polarization [see the dotted $(\Omega / \Gamma=1)$ and dashdotted $(\Omega / \Gamma=5)$ lines in Fig. $3(\mathrm{c})]$.

Let us now turn to the situation where the electrodes are of AP alignment. The self-correlations $S_{\alpha \alpha}^{\uparrow \uparrow}$ and $S_{\alpha \alpha}^{\downarrow \downarrow}$ vs spin polarization $p$ are plotted in Fig.5(a) and (b), respectively. Again, we take $S_{\alpha \alpha}^{\uparrow \uparrow}$ for illustration. If the left electrode is fully spin-down polarized, transport of up spins are completely suppressed, which leads to a vanishing $S_{\alpha \alpha}^{\uparrow \uparrow}$ as $p \rightarrow-1$. In the opposite limit of $p \rightarrow 1$, only up spins are allowed to tunnel into the coupled dots; however, under the AP alignment the rate of tunneling out to the right electrode is strongly inhibited. In this case, tunneling of up spins is in close analogy to electron tunneling through an extremely asymmetric double barrier structure [3]. The up spin tunneling events are thus uncorrelated, and the resultant noise correlation turns out to be Poisson $\left(S_{\alpha \alpha}^{\uparrow \uparrow} \rightarrow 1\right)$, independent of interdot coupling strength $\Omega$. In a wide range in between, the noise is very sensitive to the interdot coupling strength. Particularly, we observe a super-Poissonian spin self-correlation $S_{\alpha \alpha}^{\uparrow \uparrow}$, as shown by the solid line in Fig. 5 (a). The occurrence of dynamical spin $\uparrow-\downarrow$ bunching relies on two conditions: (i) Appropriate spin polarization in the electrodes, and (ii) strong localization of a down spin in QD2, which is fulfilled at weak interdot coupling $\Omega\left(\Omega<\frac{1}{9} \Gamma\right.$ according to our calculation). A rising interdot coupling leads to delocalization of the down spin. The dynamical spin $\uparrow-\downarrow$ blockade is lifted, which results eventually in a subPoisson self-correlation for arbitrary polarization [see, for instance, the dashed line in Fig. [5(a) for $\Omega / \Gamma=0.2]$.

Although neither of the two spin self-correlations $\left(S_{\alpha \alpha}^{\uparrow \uparrow}\right.$ and $S_{\alpha \alpha}^{\downarrow \downarrow}$ ) exhibits super-Poisson statistics for $\Omega / \Gamma=0.2$, the total charge current noise displays unambiguously super-Poisson characteristics [dashed line in Fig.[5(d)]. Analogous to situation of $\mathrm{P}$ configuration, the superPoisson noise here arises from the dynamical spin $\uparrow-\uparrow$ or $\downarrow-\downarrow$ bunching, as one can infer from the positive spin mutual-correlation [dashed line in Fig.[5(c)]. A decrease in the strength of interdot tunnel-coupling leads to enhancement of the dynamical spin $\uparrow-\uparrow$ or $\downarrow-\downarrow$ bunching, and finally increases the spin mutual-correlation [cf. Fig. Thus, if the interdot tunnel-coupling is low enough one may observe the coexistence of dynamical spin $\uparrow-\downarrow(\downarrow-\uparrow)$ and $\uparrow-\uparrow(\downarrow-\downarrow)$ bunching of tunneling events, as indicated by the super-Poisson spin self-correlation and positive mutual-correlation Fig. (5 (a) and (b), respectively. Yet, different from the $\mathrm{P}$ alignment, the dynamical spin $\uparrow-\uparrow$ blockade survives even for a strong interdot coupling $\Omega$, as long as the electrodes are not weakly polarized. In this case, an up spin injected into the double dots may experience some oscillations between the two dots before it can tunnel out to the right electrode owing to a small $\Gamma_{\mathrm{R} \uparrow}$. Its dwell on the double dots serves an "effective" dynamical spin $\uparrow-\uparrow$ blockade mechanism, yielding thus a positive spin mutual-correlation. On the other hand, the small $\Gamma_{\mathrm{R} \uparrow}$ also inhibits tunneling of up spins, which explains the suppressed spin mutual-correlation observed in Fig. 馬(c).

We note that current transport through similar structure has also been investigated recently in [31, 53] and super-Poissonian noise was reported. There, the existence of a very lower tunneling rate between QD2 and the electrodes than that between QD1 and the electrodes leads to finite occupation of the QD2, which gives rise eventually to the super-Poissonian fluctuations. Although the mechanisms are different, the finally results happen to be consistent qualitatively. That is, the total charge current noise is larger in the $\mathrm{P}$ configuration than in the AP one for a large polarization, see Fig.3(d) and Fig. 5 (d).

\section{CONCLUSION}

In the context of spin-dependent transport though a Coulomb blockaded double quantum dot system, we revealed unambiguously unique dynamical spin $\uparrow-\uparrow$ and $\downarrow-\downarrow$ 
bunching of tunneling events, as confirmed by the realtime Monte Carlo simulation of spin tunneling. Different from the conventional dynamical spin $\uparrow-\downarrow$ bunching, this new mechanism is found to be intimately associated with the spin mutual-correlation for both (parallel and antiparallel) magnetic configurations. Under conditions of low spin polarization and weak interdot tunnel-coupling, we demonstrated the coexistence of the dynamical spin $\uparrow-\downarrow$ and $\uparrow-\uparrow$ bunching events. Our analysis together with recent suggestions on measurement of spin-resolved noise correlations [54 56] may shed light on possibilities towards feedback control of spin transport through quan- tum dot systems [57, 58].

\section{Acknowledgments}

This work was supported by the National Natural Science Foundation of China (grant nos 11204272, 11147114, and 11004124) and the Zhejiang Provincial Natural Science Foundation (grant nos Y6100171, Y6110467, and LY12A04008).
[1] Y. M. Blanter and M. Büttiker, Phys. Rep. 336, 1 (2000).

[2] Y. V. Nazarov, Quantum Noise in Mesoscopic Physics (Kluwer, Dordrecht, 2003).

[3] L. Y. Chen and C. S. Ting, Phys. Rev. B 43, 4534 (1991).

[4] W. D. Oliver, J. Kim, R. C. Liu, and Y. Yamamoto, Science 284, 299 (1999).

[5] M. Henny, S. Oberholzer, C. Strunk, T. Heinzel, K. Ensslin, M. Holland, and C. Schönenberger, Science 284, 296 (1999).

[6] W. G. van der Wiel, S. D. Franceschi, J. M. Elzerman, T. Fujisawa, S. Tarucha, and L. P. Kouwenhoven, Rev. Mod. Phys. 75, 1 (2003).

[7] G. Kießlich, E. Schöll, T. Brandes, F. Hohls, and R. J. Haug, Phys. Rev. Lett. 99, 206602 (2007).

[8] S. Lindebaum, D. Urban, and J. König, Phys. Rev. B 79, 245303 (2009).

[9] G. Michałek and B. R. Bułka, Phys. Rev. B 80, 035320 (2009).

[10] J. Y. Luo, H. J. Jiao, Y. Shen, G. Cen, X.-L. He, and C. Wang, J. Phys.: Condens. Matter 23, 145301 (2011).

[11] J. Y. Luo, Y. Shen, X.-L. He, X.-Q. Li, and Y. J. Yan, Phys. Lett. A 376, 59 (2011).

[12] N. Lambert and F. Nori, Phys. Rev. B 78, 214302 (2008).

[13] G. A. Prinz, Science 282, 1660 (1998).

[14] S. A. Wolf, D. D. Awschalom, R. A. Buhrman, J. M. Daughton, S. von Motnár, M. L. Roukes, A. Y. Chtchelkanova, and D. M. Treger, Science 294, 1488 (2001).

[15] F. J. Jedema, A. T. Filip, and B. J. van Wees, Nature 410, 345 (2001).

[16] F. J. Jedema, H. B. Heersche, A. T. Filip, J. J. A. Baselmans, and B. J. van Wees, Nature 416, 713 (2002).

[17] D. D. Awschalom, D. Loss, and N. Samarth, Semiconductor spintronics and quantum computation (Springer, Berlin, 2002).

[18] J. J. L. Morton, D. R. McCamey, M. A. Eriksson, and S. A. Lyon, Nature 479, 345 (2011).

[19] R. Hanson, L. P. Kouwenhoven, J. R. Petta, S. Tarucha, and L. M. K. Vandersypen, Rev. Mod. Phys. 79, 1217 (2007).

[20] I. Z̆utić, J. Fabian, and S. D. Sarma, Rev. Mod. Phys. 76, 323 (2004).

[21] A. Thielmann, M. H. Hettler, J. König, and G. Schön, Phys. Rev. Lett. 95, 146806 (2005).

[22] M. Braun, J. König, and J. Martinek, Phys. Rev. B 74, 075328 (2006).
[23] I. Weymann, J. Barnaś, and S. Krompiewski, Phys. Rev. B 85, 205306 (2012).

[24] H. Yu and J.-Q. Liang, Phys. Rev. B 72, 075351 (2005).

[25] M. Misiorny, I. Weymann, and J. Barnaś, Phys. Rev. B 79, 224420 (2009).

[26] I. Weymann and J. Barnaś, Phys. Rev. B 82, 165450 (2010).

[27] I. Weymann, J. Barnaś, and S. Krompiewski, Phys. Rev. B 78, 035422 (2008).

[28] S. Lipiński and D. Krychowski, Phys. Rev. B 81, 115327 (2010).

[29] F. Wu, P. Queipo, A. Nasibulin, T. Tsuneta, T. H. Wang, E. Kauppinen, and P. J. Hakonen, Phys. Rev. Lett. 99, 156803 (2007).

[30] I. Weymann, B. R. Bułka, and J. Barnaś, Phys. Rev. B 83, 195302 (2011).

[31] I. Weymann and J. Barnaś, Phys. Rev. B 77, 075305 (2008).

[32] A. Cottet, W. Belzig, and C. Bruder, Phys. Rev. B 70, 115315 (2004).

[33] A. Cottet, W. Belzig, and C. Bruder, Phys. Rev. Lett. 92, 206801 (2004).

[34] B. Dong, X. L. Lei, and N. J. M. Horing, Phys. Rev. B 80, 153305 (2009).

[35] A. Nauen, I. Hapke-Wurst, F. Hohls, U. Zeitler, R. J. Haug, and K. Pierz, Phys. Rev. B 66, 161303 (2002).

[36] S. S. Safonov, A. K. Savchenko, D. A. Bagrets, O. N. Jouravlev, Y. V. Nazarov, E. H. Linfield, and D. A. Ritchie, Phys. Rev. Lett. 91, 136801 (2003).

[37] A. Nauen, F. Hohls, J. Könemann, and R. J. Haug, Phys. Rev. B 69, 113316 (2004).

[38] S. Sasaki, H. Tamura, T. Akazaki, and T. Fujisawa, Phys. Rev. Lett. 103, 266806 (2009).

[39] T.-S. Kimand and S. Hershfield, Phys. Rev. B 63, 245326 (2001).

[40] P. S. Cornaglia and D. R. Grempel, Phys. Rev. B 71, 075305 (2005).

[41] I. Djuric, B. Dong, and H. L. Cui, Appl. Phys. Lett. 87, 032105 (2005).

[42] J. Y. Luo, S.-K. Wang, X.-L. He, X.-Q. Li, and Y. J. Yan, J. Appl. Phys. 108, 083720 (2010).

[43] J. Y. Luo, X.-Q. Li, and Y. J. Yan, Phys. Rev. B 76, $085325(2007)$

[44] J. Y. Luo, X.-Q. Li, and Y. J. Yan, J. Phys.: Cond. Matt. 20, 345215 (2008).

[45] K. Ono, D. G. Austing, Y. Tokura, and S. Tarucha, Sci- 
ence 297, 1313 (2002)

[46] T. Fujisawa, T. Hayashi, R. Tomita, and Y. Hirayama, Science 312, 1634 (2006).

[47] F. H. L. Koppens, C. Buizert, K. J. Tielrooij, I. T. Vink, K. C. Nowack, T. Meunier, L. P. Kouwenhoven, and L. M. K. Vandersypen, Nature 442, 766 (2006).

[48] H. S. Goan, G. J. Milburn, H. M. Wiseman, and H. B. Sun, Phys. Rev. B 63, 125326 (2001).

[49] H. S. Goan and G. J. Milburn, Phys. Rev. B 64, 235307 (2001).

[50] S. A. Gurvitz, D. Mozyrsky, and G. P. Berman, Phys. Rev. B 72, 205341 (2005).

[51] I. Djuric and C. P. Search, Phys. Rev. B 74, 115327 (2006).
[52] D. A. Bagrets and Y. V. Nazarov, Phys. Rev. B 67, 085316 (2003).

[53] I. Weymann, Phys. Rev. B 78, 045310 (2008).

[54] O. Sauret and D. Feinberg, Phys. Rev. Lett. 92, 106601 (2004).

[55] J. Foros, A. Brataas, Y. Tserkovnyak, and G. E. Bauer, Phys. Rev. Lett. 95, 016601 (2005).

[56] M. Covington, M. AlHajDarwish, Y. Ding, N. J. Gokemeijer, and M. A. Seigler, Phys. Rev. B 69, 184406 (2004).

[57] T. Brandes, Phys. Rev. Lett. 105, 060602 (2010).

[58] G. Kießlich, C. Emary, G. Schaller, and T. Brandes, LANL e-print arXiv:1209.1226 (2012). 